\title{
Lift-Off Instability During the Impact of a Drop on a Solid Surface
}

\section{Citation}

Kolinski, John M., L. Mahadevan, and Shmuel M. Rubinstein. 2014. "Lift-Off Instability During the Impact of a Drop on a Solid Surface." Physical Review Letters 112 (13). https://doi.org/10.1103/ physrevlett.112.134501.

\section{Permanent link}

http://nrs.harvard.edu/urn-3:HUL.InstRepos:41412231

\section{Terms of Use}

This article was downloaded from Harvard University's DASH repository, and is made available under the terms and conditions applicable to Other Posted Material, as set forth at http:// nrs.harvard.edu/urn-3:HUL.InstRepos:dash.current.terms-of-use\#LAA

\section{Share Your Story}

The Harvard community has made this article openly available.

Please share how this access benefits you. Submit a story.

Accessibility 


\title{
Lift-Off Instability During the Impact of a Drop on a Solid Surface
}

\author{
John M. Kolinski, ${ }^{1,2}$ L. Mahadevan, ${ }^{1,3}$ and Shmuel M. Rubinstein ${ }^{1,2, *}$ \\ ${ }^{1}$ School of Engineering and Applied Sciences, Harvard University, Cambridge, Massachusetts 02138, USA \\ ${ }^{2}$ Department of Physics of Complex Systems, Weizmann Institute of Science, Rehovot 76100, Israel \\ ${ }^{3}$ Department of Physics, Harvard University, Cambridge, Massachusetts 02138, USA \\ (Received 15 August 2013; revised manuscript received 16 December 2013; published 4 April 2014)
}

\begin{abstract}
We directly measure the rapid spreading dynamics succeeding the impact of a droplet of fluid on a solid, dry surface. Upon impact, the air separating the liquid from the solid surface fails to drain and wetting is delayed as the liquid rapidly spreads outwards over a nanometer thin film of air. We show that the approach of the spreading liquid front toward the surface is unstable and the spreading front lifts off away from the surface. Lift-off ensues well before the liquid contacts the surface, in contrast with prevailing paradigm where lift-off of the liquid is contingent on solid-liquid contact and the formation of a viscous boundary layer. Here we investigate the dynamics of liquid spreading over a thin film of air and its lift-off away from the surface over a large range of fluid viscosities and find that the lift-off instability is dependent on viscosity and occurs at a time that scales with the viscosity to the power of one half.
\end{abstract}

DOI: $10.1103 /$ PhysRevLett.112.134501

PACS numbers: 47.55.D-, 47.55.N-, 68.03.-g

Over a century since the pioneering work of Worthington investigating splashing of liquid drops [1], the dynamics of the interaction between an impacting drop of fluid and the surface it wets has attracted the attention of researchers [1-8]. When drop impact occurs at moderate velocities, the dynamics are traditionally considered to be quite simple: For a drop that is brought into contact at a slow rate, contact initiates at a point centered on the impact axis, then spreads laterally to coat the surface uniformly $[9,10]$. The rate at which the contact line spreads is determined by the balance between the inertia of the liquid and the surface tension of the interface $[9,10]$. In these regimes, viscosity is assumed to be negligible in comparison to inertia and surface tension and its effect is therefore ignored [9,10]. Since the dynamics of this slow approach are governed by inertia, it is customary to sustain this picture when the approach velocity is increased $[11,12]$. The model for slow approach, however, completely ignores the role that the surrounding air plays in the impact dynamics. Indeed, before a drop will contact a surface, the separating air must first be drained. Draining the air becomes more difficult as the gap between the liquid and the solid surface diminishes, up to a time when the air fails to drain and instead compresses, while the bottom surface of the drop is deformed and the liquid spreads laterally outward and not downward. As a result of this process, a small bubble of air remains trapped within the liquid once contact occurs [13-17]. Indeed, many beautiful experiments have shown over the past few years that the ambient air has a critical role in the dynamics of droplet impact [6,15,18-28]. Perhaps the most striking example for the role of air in the dynamics of impacting drops is the total suppression of corona splashing when the ambient atmospheric pressure is reduced to a third of an atmosphere [6]. Corona splashing is characterized by the ejection of a thin liquid sheet and its subsequent uplift and eventual breakup into droplets during droplet impact [22]. More recently, it was shown, initially theoretically $[29,30]$ and then experimentally [27], that when a drop impacts a surface the outward spreading of the liquid can occur over a thin film of air, a few hundreds of nanometers in thickness or even less. Indeed, the air film thickness can be even less than the mean free path of air at room temperature and pressure. However, the gas can be significantly compressed beneath the liquid as it impacts upon the surface and the mean free path of the air reduced, thereby averting noncontinuum effects such as enhanced slip at the boundary [31]. The presence of a thin lubricating air layer enables the liquid to spread outward at very high velocities, high enough to support the formation of a singular sheet of liquid at the leading edge. However, the mechanism for the lift-off of the liquid away from the surface that enables splashing at high impact velocities remains elusive. Current theoretical models and calculations require the initiation of full contact between the fluid and solid [31]; this in turn requires the formation of a viscous boundary layer to enable lift-off of the liquid. Experimental testing of these dynamics is challenging and requires ultrafast measurement of the nanometer-thin film of air. In this Letter we explore in detail the fluid dynamics occurring as drops of Newtonian liquids impact on a solid, dry surface. We investigate the rapid spreading of the liquid over a nanometer-thin film of air following the impact of the drop for water-glycerol mixtures and for nonaqueous silicone liquids over a wide range of viscosities. The surface of impact is imaged from below with rapid Total Internal Reflection (TIR) microscopy [27]. We find that the initial spreading velocities of the liquid are largely independent of the viscosity of the liquid. This observation is consistent with 

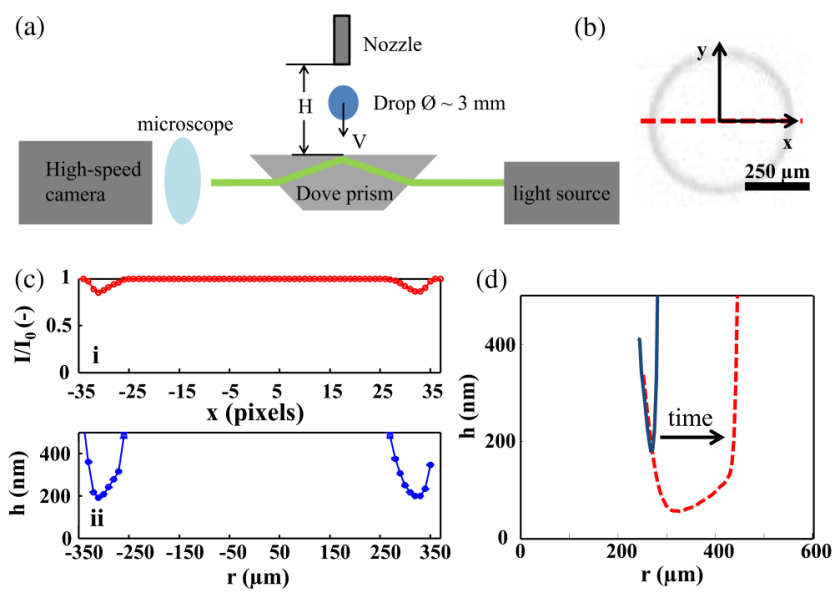

FIG. 1 (color online). TIR microscopy: (a) Schematic of the experimental setup. (b) A typical fast camera snapshot of a liquid-air interface before contact occurs taken approximately 10 microseconds after the dimple has formed. (c) (i) The normalized intensity trace taken along the cut marked by the red dashed line in (b). (ii) The height of the liquid above the solid surface plotted against distance for the same trace shown in (c). (d) Two sample axisymmetric liquid-air interface profiles, demonstrating the transition from a sharp cusp (dark solid line) to the curved, rounded profile (dashed line), as the liquid-air interface transitions to lifting off away from the surface.

theoretical predictions, which showed that the dynamics of drop impact are approximated well by an inviscid liquid [29]. Surprisingly, we also observe a new instability in the profile of the spreading liquid which was not previously observed nor predicted theoretically; the leading edge of the liquid abruptly transitions from an extremely sharp cusp into a curved, rounded profile and the liquid subsequently lifts off away from the surface, as shown in Fig. 1(d). It is interesting that although the spreading rate is independent of the liquid viscosity, the time at which the transition to lift-off occurs relative to the instant of initial impact does depend on viscosity, and scales as the viscosity to the power of $1 / 2$. The rapid dynamics occurring directly above the interface are measured with TIR microscopy and a fast camera. TIR is a well-established imaging technique that we recently adapted for fluid dynamics, and which enables us to directly probe the dynamics of nanometer thin films of air formed beneath the liquid drop with unprecedented high speeds. The experimental setup is described elsewhere [27], and is also shown schematically in Fig. 1(a). A collimated, monochromatic beam of light undergoes total internal reflection off of the surface of impact [32], generating an exponentially decaying evanescent field. The angle of incidence of the light on the interface is chosen such that the condition for total internal reflection is maintained for a glass-air interface, but not for the glass-liquid interface. The reflected intensity is imaged with our fast camera's sensor. When a drop of liquid enters the evanescent field, light tunnels through the liquid-air interface and the reflected intensity decreases; this appears as a gray scale on our imaging sensor. The evanescent wave decays over a length scale which is a function of the angle of incidence, and in our experiments is typically $100 \mathrm{~nm}$, allowing us to clearly identify films of air as thin as a few nanometers. The high resolution achieved by TIR allows us to directly observe the thin film of air that initially appears as a gray ring on our imaging sensor, as shown in Fig. 1(b). We are also able to sharply distinguish between surfaces that are separated from the liquid by a thin film of air and a wet surface, as well as extracting absolute height information by converting the pixel gray scale intensity into height, as shown in Fig. 1(c). To investigate the initial impact dynamics of drops over a large range of parameters, we study drops of different size and surface tension, and vary the viscosity by two orders of magnitude, from 1 to $100 \mathrm{cSt}$. This is obtained by using both waterglycerol mixtures and silicone oils of various viscosities. We restrict this study to initial release heights, $H$, between $8 \mathrm{~mm}$ and $30 \mathrm{~mm}$ and image the dynamics at a rate of up to 180,000 frames per second with a fast camera (Phantom V711). We focus on this velocity regime in order to decouple the dynamics of the liquid-air interface from the dynamics of liquid-solid contact, described elsewhere [27]. Before the liquid contacts the solid, the air beneath the drop flows out but fails to completely drain, and the bottom surface of the drop deforms as the liquid funnels outwards; this process leads to the formation of a dimple that at later times develops into a trapped bubble of air within the drop [15,28]. Moreover, after forming the dimple, the liquid does not immediately wet the surface, but instead continues skating rapidly over a nm-thin film of air, as shown for an aqueous drop impacting a smooth glass surface at an impact speed $V=0.45 \mathrm{~m} / \mathrm{sec}$, in Fig. 2(a). In all of our experiments, we observe the liquid skating over a nm-thin film, consistent with previous experiments conducted with a low-viscosity alcohol [27]; moreover, for all the liquids we used, the initiation of liquid-solid contact in each of our experiments occurred similarly to previously observed breakdown of the thin air film [27]. In this letter we restrict our description to the axisymmetric dynamics occurring prior to any wetting. For a given impact velocity, the initial rate at which the liquid spreads over the thin film of air is nearly identical for all the different liquids, in spite of a difference of two orders of magnitude in viscosity. This can be seen by the similar spacing between the time-dependent profiles shown in Fig. 2(b) and (c) as well as also in the inset to Fig. 3(b) where we plot the instantaneous spreading rates of liquids of three different viscosities. In spite of the striking consistency in spreading rates, the spreading dynamics do indeed vary markedly for different viscosities. For all impact parameters measured the leading edge of the liquid initially progresses slightly toward the surface; however, this process is unstable. Instead, a sharp transition is observed, with the liquid abruptly lifting off away from the surface. While for low-viscosity liquids the lift-off occurs almost immediately, for the higher 
(a)

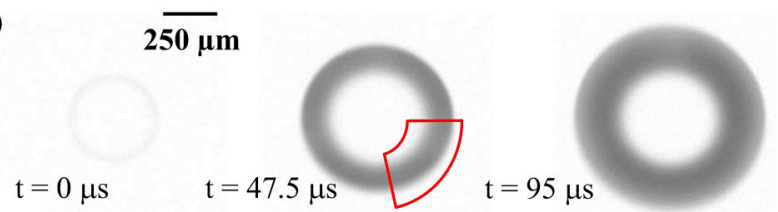

(b)
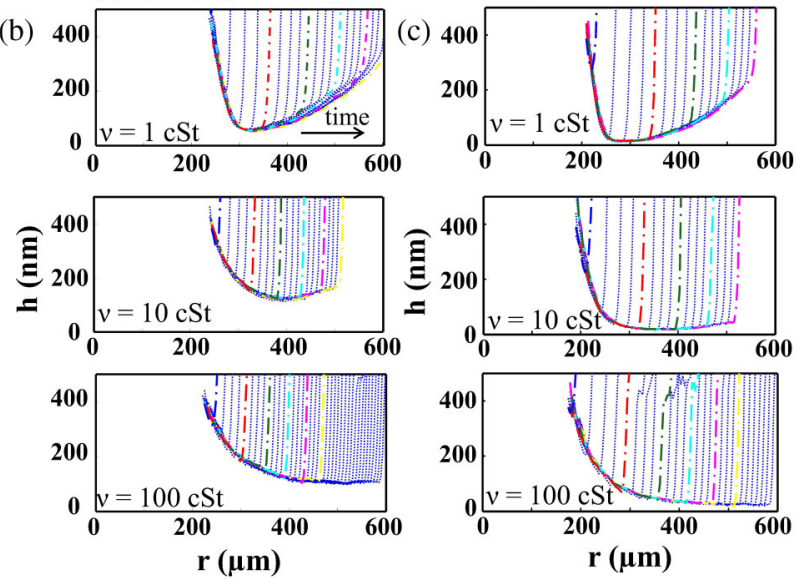

FIG. 2 (color online). Skating on a thin film of air: (a) TIR snapshots taken at $2 \mu \mathrm{sec}$ exposure, showing the spreading of a $10 \mathrm{cSt}$ water-glycerol mixture over a thin film of air. The red semiannular region denotes a typical area used to calculate the annular-average profiles shown in (b) and (c). (b) Liquid-air interface profiles for water-glycerol mixtures with three different viscosities, impacting at $V=0.45 \mathrm{~m} / \mathrm{sec}$. The different curves within each plot are separated by $\sim 5.5 \mu$ sec. (c) Profiles for impacting drops of identical dimensions to those in (b), and $V=0.63 \mathrm{~m} / \mathrm{sec}$. The asymptotic air film thickness is on average 4 times smaller than in (b); nevertheless, for both impact velocities, the dependence of the spreading dynamics on the liquid viscosity is qualitatively similar. Color is used to highlight traces occurring at the same time.

viscosity liquids it is delayed for up to a hundred microseconds during which the liquid spreads to a lateral extent exceeding several hundred microns, as shown in Fig. 2(b) and (c). In fact, for the $100 \mathrm{cSt}$ liquid, the air layer is already beginning to break down beneath the liquid before we observe a liftoff of the spreading front. Surprisingly, even though the thickness of the thin film of air significantly decreases with impact velocity, all other features of the spreading dynamics are remarkably similar; thus, they depend strongly on the viscosity of the liquid and not significantly on the thickness of the thin film of air beneath the spreading liquid, as shown by comparing the two panels in Fig. 2(b) and (c).

We characterize the viscosity dependence of the spreading and lift-off dynamics by examining individual profiles. A typical example of the height profile, $h(r)$, of a low viscosity liquid spreading over a thin film of air is shown in Fig. 3(a). For each experiment, we identify two points on the profile of the thin film of air, one where the liquid is closest to the surface, whose distance from the impact center is $r_{m}$ and whose height above the surface is $h_{m}$, as indicated by the red circle in Fig. 3(a). The second point is
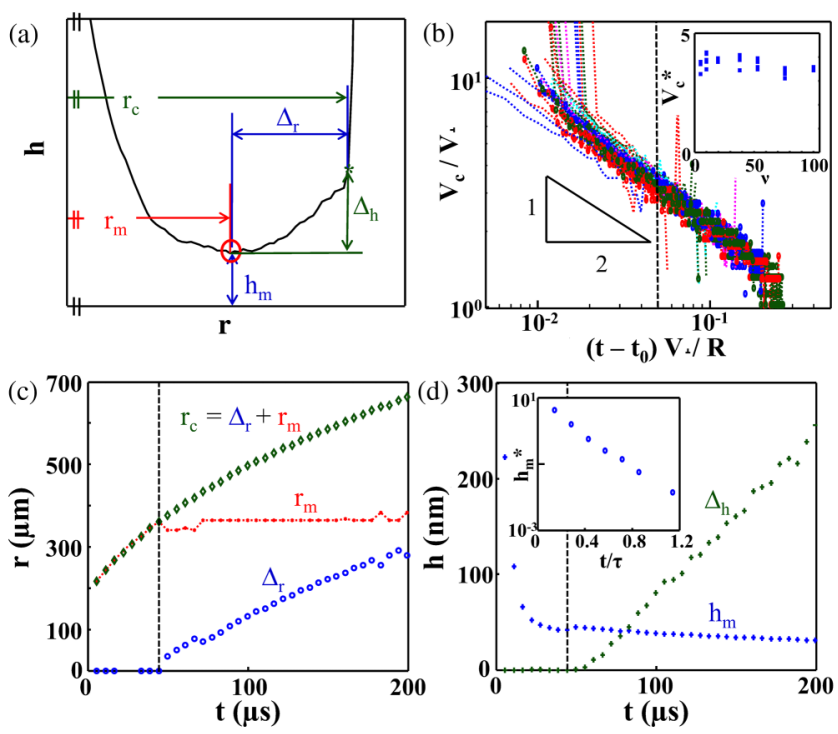

FIG. 3 (color online). Spreading and lift-off dynamics. (a) Typical example of the profile, $h(r)$ of a low viscosity liquid spreading over a thin film of air. (b) Normalized spreading velocity parallel to the surface, $V_{c} / V$ plotted against time normalized by the impact time scale, $\left(t-t_{0}\right) V_{\perp} / R$. The curves collapse to one master curve for $V=0.45 \mathrm{~m} / \mathrm{sec}$ to $V=0.63 \mathrm{~m} / \mathrm{sec}$. (Insert) $V_{c}^{*}$, for a range of liquid viscosities. (c) A typical example of the spreading dynamics $r(t)$ shown for a $10 \mathrm{cSt}$ impacting at $0.64 \mathrm{~m} / \mathrm{sec}$. The time $\tau$ when the leading edge of the drop $r_{c}$ begins to differ from the point closest to the surface $r_{m}$ is precisely where the liquid lifts off. (d) $h_{m}$ and $h_{c}$ as a function of time for the same experiment plotted in (c). (Insert) A semilogarithmic plot of $h_{m}^{*}=\left(h_{m}(t)-h_{m}(\tau)\right) / h_{m}(\tau)$ plotted as a function of $t / \tau$. Note that the liquid approaches the surface at an exponential rate.

where the liquid front exhibits maximal curvature, which is a good measure for the location of the leading edge of the liquid. Its radial distance to the drop impact center is $r_{c}$ and its height above the surface is $h_{c}$, indicated by a green asterisk in Fig. 3(a). We calculate the rate at which the liquid spreads outward, $V_{c}$, by taking the numerical derivative of $r_{c}$. The outward spreading of the liquid sets in as the drop approaches the surface immediately following its deformation by the compressed air; therefore, the liquid is funneled outward at a velocity that decays as $t^{-1 / 2}$ [10], as shown in Fig. 3(b). In the initial stages of impact, the spreading is dominated by the inertia of the drop and the spreading velocity is predominantly independent of viscosity, as shown in the inset to Fig. 3(b) where we compare the instantaneous spreading velocity, $V_{c}^{*}$, measured at a normalized time $t V / R=0.05$, marked by the dashed line in the main figure. The origin of $t, t_{0}$ is the estimated instant that the center of the drop would contact the surface in the absence of air, $t_{0}=r_{m 0}^{2} / 2 R V$, where $r_{m 0}$ is defined as $r_{m}$ at the first instant that the liquid enters the evanescent field. The outward spreading front progresses at a rate independent of liquid viscosity, nevertheless, the dynamics of the lift-off away from the surface do depend strongly on liquid 

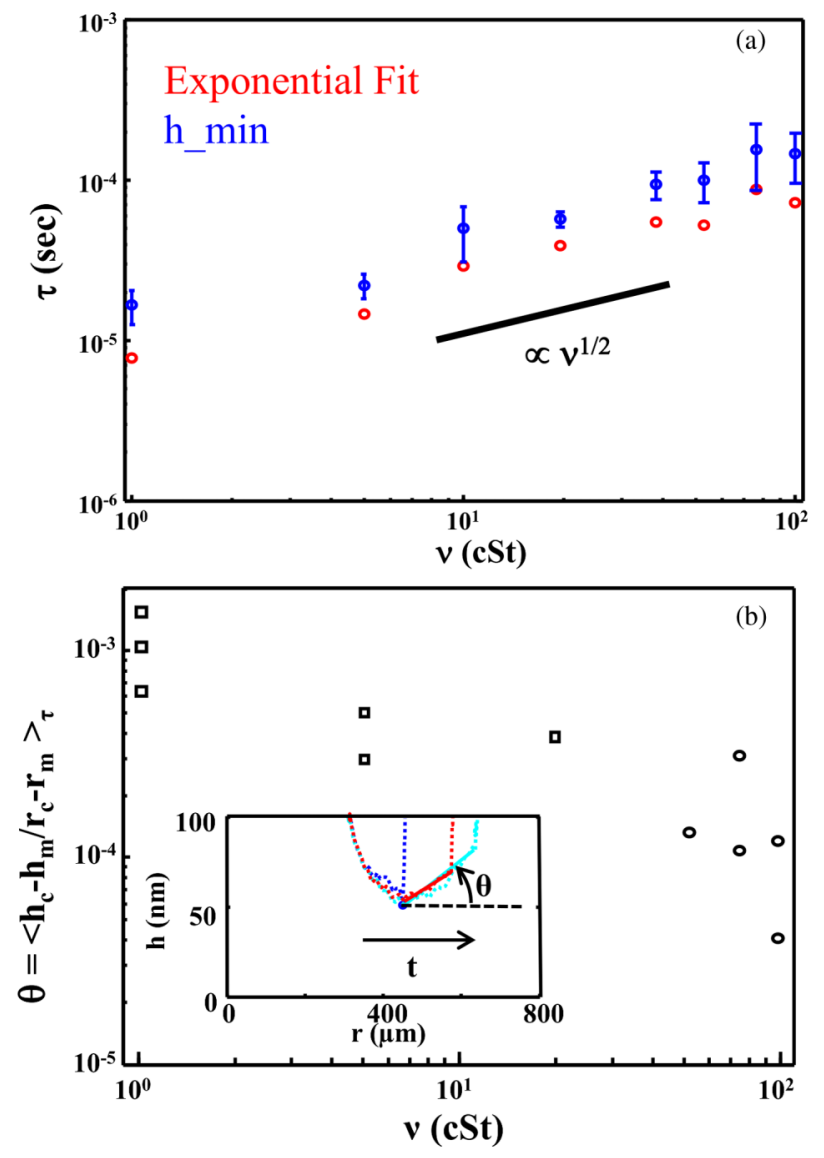

FIG. 4 (color online). Lift-off dynamics: (a) Typical time scale before lift-off, $\tau$, as a function of viscosity, $\nu$, measured in two different ways: extracting a typical time scale from an exponential fit to $h_{\min }(t)$ (red) and the first instance $\Delta_{r}>0$ (blue). The black line is a $\nu^{1 / 2}$ and serves as a guide for the eye (b) Lift-off angle, $\Theta$, highlighted in the inset and as a function of $\nu$.

viscosity. For all viscosities the liquid front is initially led by a sharp, high-curvature cusp and the fastest spreading liquid is closest to the surface; thus, at this stage, $r_{m}=r_{c}$. However, after a time $\tau$ that does depend on viscosity, the liquid lifts off away from the surface, as highlighted by the dashed black line in Fig. 3(c); thus, $r_{c}$ deviates from $r_{m}$ and $h_{c}$ differs from $h_{m}$. We define these parting length scales as $\Delta_{r}$ and $\Delta_{h}$ respectively. At time $\tau, \Delta_{r}$ and $\Delta_{h}$ sharply increase from zero, as clearly seen for $\Delta_{r}$ in Fig. 3(c) and $\Delta_{h}$ in Fig. 3(d), respectively. $h_{m}$ decreases exponentially as shown by the inset in Fig. 3(d). Surprisingly, before $h_{m}$ decays to an asymptotic value, $r_{m}$ stops increasing abruptly; this corresponds to the moment the spreading front begins to lift-off away from the surface. The time $\tau$ at which the fluid motion transitions to lift away from the surface is measured relative to the initial entry of the liquid into the evanescent field, and highlighted by the vertical dashed lines in Fig. 3(c) and (d). The time scale $\tau$ corresponding to the sudden lift-off transition exhibits a scaling of $\nu^{1 / 2}$, as shown in Fig. 4(a). Since the velocity of spreading is independent of viscosity, our results indicate that the point of lift-off is significantly farther from the impact center for more viscous fluids compared to less viscous fluids. This is perhaps analogous to recent results showing that viscous drops delay ejection of a sheet during a splash [22]. Moreover, we observe a persistent slope once the liquid begins to lift off away from the surface. The lift-off is much flatter for more viscous drops, as shown by the average slope shown in Fig. 4(b), which may also be analogous to the flatter corona sheet observed in the viscous splashing experiments [22]. However, those viscous splashing experiments [22] are conducted with considerably higher impact velocities; furthermore, in those and similar experiments, no persistent thin film of air beneath the impacting drop was observed. As impact velocity increases beyond the range of velocities we investigate in this work, out temporal resolution is no longer sufficient to observe the lift-off transition through the wetting dynamics. Nevertheless, the similarity between the behaviors in these two experimental regimes may suggest that the instability leading to the lift-off of the spreading front is related to the mechanism for the formation and rise of the corona in a viscous splash. However, determining whether or not the novel lift-off dynamics reported here are directly related to splashing will require future studies of high speed droplet impact.

Research supported by ISF Grant No. 1415/12. J. M. K. acknowledges the support from the NDSEG and NSF Graduate Fellowships.

* Corresponding author.

shmuel@seas.harvard.edu

[1] A. Worthington, Proc. R. Soc. London 25, 261 (1876).

[2] M. Lesser, Proc. R. Soc. A 377, 289 (1981).

[3] M. Lesser and J. Field, Annu. Rev. Fluid Mech. 15, 97 (1983).

[4] I. V. Roisman, R. Rioboo, and C. Tropea, Proc. R. Soc. A 458, 1411 (2002).

[5] A. Lafuma and D. Quéré, Nat. Mater. 2, 457 (2003).

[6] L. Xu, W. W. Zhang, and S. R. Nagel, Phys. Rev. Lett. 94, 184505 (2005).

[7] A. Yarin, Annu. Rev. Fluid Mech. 38, 159 (2006).

[8] J. de Ruiter, R. E. Pepper, and H. A. Stone, Phys. Fluids 22, 022104 (2010).

[9] A.-L. Biance, C. Clanet, and D. Quéré, Phys. Rev. E 69, 016301 (2004).

[10] J. C. Bird, S. Mandre, and H. A. Stone, Phys. Rev. Lett. 100, 234501 (2008)

[11] A. L. Moreira, A. Moita, and S. Chandra, in Handbook of Atomization and Sprays (Springer, New York, 2011), p. 183.

[12] R. D. Schroll, C. Josserand, S. Zaleski, and W. W. Zhang, Phys. Rev. Lett. 104, 034504 (2010).

[13] S. Thoroddsen and J. Sakakibara, Phys. Fluids 10, 1359 (1998).

[14] S. Thoroddsen, T. Etoh, and K. Takehara, J. Fluid Mech. 478, 125 (2003).

[15] S. Thoroddsen, T. Etoh, K. Takehara, N. Ootsuka, and Y. Hatsuki, J. Fluid Mech. 545, 203 (2005). 
[16] V. Mehdi-Nejad, J. Mostaghimi, and S. Chandra, Phys. Fluids 15, 173 (2003).

[17] D. B. van Dam and C. Le Clerc, Phys. Fluids 16, 3403 (2004).

[18] Y. Couder, E. Fort, C.-H. Gautier, and A. Boudaoud, Phys. Rev. Lett. 94, 177801 (2005).

[19] L. Xu, Phys. Rev. E 75, 056316 (2007).

[20] L. Xu, L. Barcos, and S. R. Nagel, Phys. Rev. E 76, 066311 (2007).

[21] P. Tsai, R. CA van der Veen, M. van de Raa, and D. Lohse, Langmuir 26, 16090 (2010).

[22] M. M. Driscoll, C. S. Stevens, and S. R. Nagel, Phys. Rev. E 82, 036302 (2010).

[23] M. M. Driscoll and S. R. Nagel, Phys. Rev. Lett. 107, 154502 (2011).

[24] A. Latka, A. Strandburg-Peshkin, M. M. Driscoll, C. S. Stevens, and S. R. Nagel, Phys. Rev. Lett. 109, 054501 (2012).

[25] J. S. Lee, B. M. Weon, J. H. Je, and K. Fezzaa, Phys. Rev. Lett. 109, 204501 (2012).
[26] R. C. A. van der Veen, T. Tran, D. Lohse, and C. Sun, Phys. Rev. E 85, 026315 (2012).

[27] J. M. Kolinski, S. M. Rubinstein, S. Mandre, M. P. Brenner, D. A. Weitz, and L. Mahadevan, Phys. Rev. Lett. 108, 074503 (2012).

[28] W. Bouwhuis, R. C. A. van der Veen, T. Tran, D. L. Keij, K. G. Winkels, I. R. Peters, D. van der Meer, C. Sun, J. H. Snoeijer, and D. Lohse, Phys. Rev. Lett. 109, 264501 (2012).

[29] S. Mandre, M. Mani, and M. P. Brenner, Phys. Rev. Lett. 102, 134502 (2009).

[30] M. Mani, S. Mandre, and M. P. Brenner, J. Fluid Mech. 647, 163 (2010).

[31] S. Mandre and M. P. Brenner, J. Fluid Mech. 690, 148 (2012).

[32] A microscope slide is used for the surface of impact and is optically coupled to a BK-7 glass dove prism using microscope objective immersion oil (Zeiss Immersol $518 \mathrm{~F}$ ). Glass microscope slides have a typical rms roughness of order 1 nanometer. 